\title{
Microbial transformations of selenite by methane-oxidizing bacteria
}

\author{
Abdurrahman S. Eswayah ${ }^{1,2}$ • Thomas J. Smith ${ }^{1}$ • Andreas C. Scheinost ${ }^{3,4}$. \\ Nicole Hondow ${ }^{5}$ • Philip H. E. Gardiner ${ }^{1}$ (D)
}

Received: 23 January 2017 /Revised: 27 May 2017 / Accepted: 30 May 2017 / Published online: 23 June 2017

(C) The Author(s) 2017. This article is an open access publication

\begin{abstract}
Methane-oxidizing bacteria are well known for their role in the global methane cycle and their potential for microbial transformation of wide range of hydrocarbon and chlorinated hydrocarbon pollution. Recently, it has also emerged that methane-oxidizing bacteria interact with inorganic pollutants in the environment. Here, we report what we believe to be the first study of the interaction of pure strains of methane-oxidizing bacteria with selenite. Results indicate that the commonly used laboratory model strains of methaneoxidizing bacteria, Methylococcus capsulatus (Bath) and Methylosinus trichosporium OB3b, are both able to reduce the toxic selenite $\left(\mathrm{SeO}_{3}{ }^{2-}\right)$ but not selenate $\left(\mathrm{SeO}_{4}{ }^{2-}\right)$ to red spherical nanoparticulate elemental selenium $\left(\mathrm{Se}^{0}\right)$, which was characterized via energy-dispersive X-ray spectroscopy (EDXS), X-ray absorption near-edge structure (XANES) and extended X-ray absorption fine structure (EXAFS). The cultures also produced volatile selenium-containing species, which suggests that both strains may have an additional activity that can transform either $\mathrm{Se}^{0}$ or selenite into volatile
\end{abstract}

Electronic supplementary material The online version of this article (doi:10.1007/s00253-017-8380-8) contains supplementary material, which is available to authorized users.

Philip H. E. Gardiner

p.h.gardiner@shu.ac.uk

1 Biomolecular Sciences Research Centre, Sheffield Hallam University, Sheffield, UK

2 Biotechnology Research Centre, Tripoli, Libya

3 The Rossendorf Beamline at ESRF, F-38043 Grenoble, France

4 Institute of Resource Ecology, Helmholtz Zentrum Dresden Rossendorf, D-01328 Dresden, Germany

5 School of Chemical and Process Engineering, University of Leeds, Leeds, UK methylated forms of selenium. Transmission electron microscopy (TEM) measurements and experiments with the cell fractions cytoplasm, cell wall and cell membrane show that the nanoparticles are formed mainly on the cell wall. Collectively, these results are promising for the use of methane-oxidizing bacteria for bioremediation or suggest possible uses in the production of selenium nanoparticles for biotechnology.

Keywords Methane-oxidizing bacteria $\cdot$ Microbial transformation $\cdot$ Selenite $\cdot$ Elemental selenium . Bioremediation

\section{Introduction}

Despite its being one of the least abundant elements in the Earth's crust, selenium is key to a number of critical biochemical reactions, and in addition to which selenium and its compounds have properties that lend themselves to many agricultural, industrial, medicinal and technological applications. Currently, only about $15 \%$ of the $2700 \mathrm{t}$ of Se produced annually is recycled (Haug et al. 2007). Minute quantities of Se are essential for normal biological functions in diverse life forms, mainly as selenocysteine, a genetically encoded amino acid incorporated into the active centres of selenoenzymes including glycine reductases, formate dehydrogenases, glutathione peroxidases, iodothyronine deiodinases and thioredoxin reductases, which play key roles in prokaryotic and eukaryotic cells (Stadtman 1991; Heider and Bock 1993; Birringer et al. 2002; Shamberger 2012; Johansson et al. 2005; Patching and Gardiner 1999). However, exposure to excessive amounts of Se released into the environment by human and natural activities can pose serious health risks to humans and may be toxic to other forms of life (Birringer et al. 2002; Lenz and Lens 2009; Qin et al. 2013). 
In Se-polluted environments, the element is mainly in the form of the water-soluble and toxic oxyanions, selenite $\left(\mathrm{SeO}_{3}{ }^{2}\right.$ $\left.{ }^{-}\right)$and selenate $\left(\mathrm{SeO}_{4}{ }^{2-}\right)$ species. A variety of microorganisms are known to transform the different chemical forms of selenium, and thus play a key role in the recycling of this valuable element and in mitigating its toxicity. Nutritionally and technologically useful forms of selenium can also be produced as a result of such microbial reactions, which include reduction, methylation, oxidation and demethylation. It appears that for most environmental microorganisms, dissimilatory reduction of the Se oxyanions and methylation of the products are the preferred microbial transformation pathways (Dungan and Frankenberger 1999; Eswayah et al. 2016). Dissimilatory reduction of selenite to elemental selenium $\left(\mathrm{Se}^{0}\right)$ has been demonstrated in a wide range of bacteria under aerobic and anaerobic conditions (Switzer Blum et al. 1998; Switzer Blum et al. 2001; Bebien et al. 2001; Klonowska et al. 2005), although the potential contribution of aerobic methane-oxidizing bacteria, which are widespread in the environment, had not until recently been explored.

Aerobic methanotrophs are a diverse and ubiquitous group of bacteria within the environment that are able to grow using methane as their sole source of carbon and energy (Hanson and Hanson 1996; Smith and Murrell 2009). At the centre of biotransformation of organic pollutants by methanotrophs are the methane monoxygenases (MMOs), which naturally oxidize methane to methanol, the first step in the conversion of the former to carbon dioxide and simple one-carbon to fourcarbon compounds to form biomass. There are two forms of MMO: soluble (sMMO) found in the cytoplasm and the particulate membrane associated (pMMO). The latter is induced at high copper-to-biomass ratios. It is noteworthy that sMMO and pMMO are different in their protein components and active site metals, but more importantly substrate specificity. Not only can methanotrophs be used to produce useful chemical products on an industrial scale, but in doing so, their ability to use methane, a cheap feedstock but potent greenhouse gas, can be harnessed to reduce its global footprint. The exploitation of methanotrophs in engineered processes has been reviewed by Jiang et al. (2010) and Kalyuzhnaya et al. (2015).

Methanotrophs are capable of remediating a wide range of hydrophobic organic pollutants, and more recently, their capacity for remediating inorganic pollutants has been recognized. Certain methanotrophs have been found able to reduce chromium(VI) to the less toxic and less bioavailable chromium(III) (Al Hasin et al. 2009; Lai et al. 2016b) and to reduce mercuric ions to metallic mercury (Boden and Murrell 2011). Recently, Lai et al. have reported the bioreduction of selenate to elemental selenium using methane as the electron donor in a membrane biofilm reactor containing a microbial community including aerobic methanotrophs (Lai et al. 2016a). These results suggested that methanotrophs can power reduction of selenium species in the environment, but did not establish whether the methanotrophs in the consortium were themselves able to transform selenium species.

In this study, two cultures of the well-characterized pure methanotroph strains, Methylococcus capsulatus (Bath) and Methylosinus trichosporium $O B 3 b$, were chosen for investigation in order to establish whether the pure strains of methane-oxidizing bacteria can biotransform selenium oxyanions. In addition, sMMO-deleted mutant of $M S$. trichosporium $\mathrm{OB} 3 \mathrm{~b}$ was used to test the hypothesis that MMO may be involved directly in the reduction of the selenium oxyanions.

\section{Materials and methods}

\section{Bacterial strains and growth conditions}

The methanotrophic bacteria Mc. capsulatus (Bath) (NCIBM 11132), Ms. trichosporium OB3b (NCIMB 11131) and Ms. trichosporium SMDM (a derivative of Ms. trichosporium OB3b in which the genes encoding sMMO have been inactivated via marker exchange mutagenesis) (Borodina et al. 2007) were grown and propagated aerobically in sterile nitrate mineral salt (NMS) media (Smith and Murrell 2011) using methane (1:4 v/v in air) as the source of carbon and energy. The selenium transformation experiments were performed in $50 \mathrm{~mL}$ liquid cultures in $250-\mathrm{mL}$ conical Quickfit ${ }^{\circledR}$ flasks capped with Suba-Seals (Sigma-Aldrich) to prevent methane loss while allowing the addition and removal of material using hypodermic syringes. The $M s$. trichosporium $\mathrm{OB} 3 \mathrm{~b}$ and Mc. capsulatus (Bath) cultures were incubated at the optimum growth temperature of 30 and $45{ }^{\circ} \mathrm{C}$, respectively, on a shaker at $180 \mathrm{rpm}$ and allowed to grow to an $\mathrm{OD}_{600}$ of $0.5-0.8$. Under the conditions used in these experiments, the Mc. capsulatus (Bath) strain grows substantially more quickly than the Ms. trichosporium OB3b stain, reaching an $\mathrm{OD}_{600}$ of 0.7 typically at $24-30 \mathrm{~h}$, whereas cultures of OB3b take 50-72 h. Addition of either sodium selenite or sodium selenate (Sigma-Aldrich, Dorset, UK) to give the desired selenium concentration was done towards the end of the logarithmic growth phase. The initial selenite concentrations used were 20 and $40 \mathrm{mg} \mathrm{L}^{-1}$ for the $M c$. capsulatus (Bath) and 10 and $20 \mathrm{mg} \mathrm{L}^{-1}$ for the $M$ s. trichosporium $\mathrm{OB} 3 \mathrm{~b}$ stain, respectively. The initial selenate concentration was $10 \mathrm{mg} \mathrm{L}^{-1}$ for either strain, respectively. Selenium stock solutions of $1000 \mathrm{mg} \mathrm{L}^{-1} \mathrm{Se}$ as $\mathrm{Na}_{2} \mathrm{SeO}_{3}$ and $\mathrm{Na}_{2} \mathrm{SeO}_{4}$ were prepared and sterilized by filtration using $0.22-\mu \mathrm{m}$ syringe filter (Millex ${ }^{\circledR}-\mathrm{GP}$ ). Three controls were set up for each experiment, with bacterial inoculum, methane and the selenium species omitted, respectively. In order to determine the cellular location of the selenite-reducing activity, grown cultures (an $\mathrm{OD}_{600}$ of $0.6-0.7$ ) of both strains were 
fractionated as previously described by Smith and Foster (1995).

\section{Investigation of the role of SMMO/pMMO in the reduction selenium oxyanions}

In order to investigate the involvement of MMOs (cytoplasmic or sMMO and the membrane-bound or pMMO), $M s$. trichosporium $\mathrm{OB} 3 \mathrm{~b}$ cultures at different stages of expression of the MMOs were amended with $10 \mathrm{mg} \mathrm{L}^{-1}$ selenite and selenate, respectively. The culture of the sMMO-deleted mutant of Ms. trichosporium SMDM was amended with either selenite or selenate and then incubated under the above conditions.

\section{Quantitation of aqueous selenite and elemental selenium}

The selenite and selenate concentrations in the amended cultures were determined by using a HPLC-ICP-MS system. Aliquots $(0.5 \mathrm{~mL})$ of the amended cultures were collected at intervals and centrifuged $(11,000 \times g ; 10 \mathrm{~min}$; room temperature), to remove the cells and other debris. An aliquot of the supernatant $(20 \mu \mathrm{L})$ was injected by a PerkinElmer LC Flexar autosampler into a PerkinElmer Flexar HPLC pump attached to a Hamilton PRP-X100 column, $4.6 \times 250 \mathrm{~mm}$, and coupled to a PerkinElmer ICP-MS NexION 350X. Separation was achieved at a flow rate of $1 \mathrm{~mL} \mathrm{~min}{ }^{-1}$ using a mobile phase made up of $5 \mathrm{mmol} \mathrm{L}^{-1}$ ammonium citrate buffer containing methanol $(2 \% v / v)$ with the $\mathrm{pH}$ adjusted to 5.2 .

The pellets were analysed for elemental selenium using a method previously described by Biswas et al. (2011) with minor modifications, as follows. Before analysis, the pellets were washed twice with $1 \mathrm{~mL}$ of $1 \mathrm{M} \mathrm{NaCl}$ in order to remove non-metabolized selenite. This high concentration of $\mathrm{NaCl}$ was employed because it had been previously found to be effective in the collection of colloidal elemental sulphur (Roy and Trudinger 1970). The washed red colloidal selenium was dissolved in $1.5 \mathrm{~mL}$ of $1 \mathrm{M} \mathrm{Na}_{2} \mathrm{~S}$, and the solution centrifuged to remove bacterial cells and cell debris.

A standard calibration curve for elemental selenium was constructed using red powdered selenium (Pfaltz \& Bauer, Waterbury, USA) dissolved in $1 \mathrm{M} \mathrm{Na}_{2} \mathrm{~S}$ solution to give a $1 \mathrm{~g} \mathrm{~L}^{-1}$ stock suspension from which working standards ranging between 10 and $50 \mathrm{mg} \mathrm{L}^{-1}$ of elemental selenium were prepared. The absorbance of each of standard solutions and samples were measured at $500 \mathrm{~nm}$.

\section{Transmission electron microscope and high-angle annular dark-field scanning transmission electron microscopy measurements}

Samples of selenite-amended culture $(1.5 \mathrm{~mL})$ were pelleted by centrifugation $(11,000 \times g ; 10 \mathrm{~min}$; room temperature $)$ and washed with $0.1 \mathrm{M}$ sodium phosphate buffer ( $\mathrm{pH}$ 7.4). The specimens were then fixed in $3 \%$ glutaraldehyde in the same buffer overnight at room temperature and washed again in the same buffer. Secondary fixation was carried out in $1 \% \mathrm{w} / \mathrm{v}$ aqueous osmium tetroxide for $1 \mathrm{~h}$ at room temperature followed by the same wash step. Fixed cells were dehydrated through a graded series of ethanol dehydration steps $(75,95$ and $100 \% v / v)$ and then placed in a 50/50 $(v / v)$ mixture of $100 \%$ ethanol and $100 \%$ hexamethyldisilazane followed by $100 \%$ hexamethyldisilazane. The specimens were then allowed to air dry overnight. A small sample of the fixed sample was crushed and dispersed in methanol, with a drop placed on a holey carbon-coated copper grid (Agar Scientific). The samples were examined in an FEI Tecnai F20 field emission gun (FEG)-TEM operating at $200 \mathrm{kV}$ and fitted with a Gatan Orius SC600A CCD camera, an Oxford Instruments XMax SDD EDX detector and a high-angle annular dark-field (HAADF) scanning TEM (STEM) detector.

\section{X-ray absorption spectroscopy}

For X-ray absorption spectroscopy (XAS) examination, the cultures were grown as described above followed by supplementation with sodium selenite (final concentration of $20 \mathrm{mg} \mathrm{L}^{-1} \mathrm{Se}$ ). After the development of the red colour, the cultures were centrifuged at $11,000 \times g$ for $10 \mathrm{~min}$. The pellet was freeze dried and analysed without further treatment. Selenium K-edge X-ray absorption near-edge structure (XANES) and extended X-ray absorption fine-structure (EXAFS) spectra were collected at the Rossendorf Beamline at ESRF (Grenoble, France). The energy of the X-ray beam was tuned by a double crystal monochromator operating in channel-cut mode using a $\mathrm{Si}(111)$ crystal pair. Two rhodiumcoated mirrors were used for collimation and suppression of higher harmonics. A 13-element high-purity germanium detector (Canberra) together with a digital signal processing unit (XIA XMap) was used to measure reaction samples in fluorescence mode. Reference samples were measured in transmission mode using ionization chambers $(300 \mathrm{~mm}$, FMB Oxford) filled with $95 \% \mathrm{~N}_{2}$ and $5 \% \operatorname{Ar}\left(\mathrm{I}_{0}\right)$ and with $100 \%$ $\operatorname{Ar}\left(\mathrm{I}_{1}\right.$ and $\left.\mathrm{I}_{2}\right)$. Spectra were collected at $15 \mathrm{~K}$ using a closed cycle $\mathrm{He}$ cryostat with a large fluorescence exit window and a low vibration level (CryoVac). Photoinduced redox reactions were effectively prevented by the cold temperature, since XANES edges remained stable during short-term exposure (10 min) as well as during the EXAFS measurements which took up to $8 \mathrm{~h}$. For energy calibration, a gold foil (K-edge at $11,919 \mathrm{eV}$ ) was chosen because of its greater inertness in comparison to Se. Data in the XANES region were collected in steps of $0.5 \mathrm{eV}$, i.e. with higher resolution than the resolution of the $\mathrm{Si}(111)$ crystal at the given vertical divergence $(1.7 \mathrm{eV})$ and the broadening due to the core-hole lifetime $(2.3 \mathrm{eV})$. A comparison of single scans of the same sample 
showed an accuracy of better than $0.5 \mathrm{eV}$. Dead time correction of the fluorescence signal, energy calibration and the averaging of single scans were performed with the software package SixPack (Webb 2005). Normalization, transformation from energy into $\mathrm{k}$ space and subtraction of a spline background was performed with WinXAS using routine procedures (Ressler 1998). The EXAFS data were fit with WinXAS using theoretical backscattering amplitudes and phase shifts calculated with FEFF 8.2 (Ankudinov and Rehr 1997). This method provides a precision of $\pm 0.01 \AA$ for shell distances and a resolution of about $\pm 0.1 \AA$ for neighbouring shells. The error of coordination numbers is $\pm 25 \%$. Statistical analysis of spectra (Eigen analysis and iterative target test) was performed with the ITFA programme package (Rossberg et al. 2003).

\section{Detection of volatile selenium species}

In order to detect volatile selenium compounds, analytical standards of dimethyl selenide $\left(\mathrm{CH}_{3} \mathrm{SeCH}_{3}\right.$, DMSe) and dimethyl diselenide $\left(\mathrm{CH}_{3} \mathrm{SeSeCH}_{3}\right.$, DMDSe) (Sigma-Aldrich, Poole, UK, >99.0 and $98 \%$, respectively) were used. Since dimethyl selenenyl sulphide $\left(\mathrm{CH}_{3} \mathrm{SeSCH}_{3}, \mathrm{DMSeS}\right)$ and methylselenol $\left(\mathrm{CH}_{3} \mathrm{SeH}, \mathrm{MeSeH}\right)$ were commercially unavailable, the compounds were synthesized as described previously (Chasteen 1993) for use as standards. Cultures of $M c$. capsulatus (Bath) and Ms. trichosporium OB3b were grown as detailed above and amended with selenite (40 and $20 \mathrm{mg} \mathrm{L}^{-1}$, respectively). Flasks containing medium inoculated with the bacteria but with no $\mathrm{SeO}_{3}{ }^{2-}$ salts added were run as controls. Samples $(200 \mathrm{~mL})$ of the headspace gas were taken through a needle attached to a sorbent tube (Tenax TA/ SulfiCarb (C2-CXXX-5314), Markes International, UK) connected to a handheld pump (Easy-VOC grab sampler, Markes International, UK) after 24 and $48 \mathrm{~h}$ for Mc. capsulatus (Bath) and Ms. trichosporium OB3b, respectively. To ensure that the tubes were contamination free, before use, the sorbent tubes were preconditioned with helium at flow rate of $90 \mathrm{~mL} \mathrm{~min}{ }^{-1}$ using the following temperature programme: $15 \mathrm{~min}$ at
$100{ }^{\circ} \mathrm{C}, 15 \mathrm{~min}$ at $200{ }^{\circ} \mathrm{C}, 15 \mathrm{~min}$ at $300{ }^{\circ} \mathrm{C}$ and $15 \mathrm{~min}$ at $335^{\circ} \mathrm{C}$.

Analysis of samples was performed on a combined thermal desorption GC-MS system. The volatiles were desorbed at $250{ }^{\circ} \mathrm{C}$ and concentrated on a thermal desorber (Unity ${ }^{\circledR}$, Markes International Limited) at $-10{ }^{\circ} \mathrm{C}$ cold trap for $5 \mathrm{~min}$ (helium flow $50 \mathrm{~mL} \mathrm{~min}^{-1}$ ) and then were transferred onto the GC/MS system (7890A-GC with 5975C-MS, Agilent Technologies) equipped with a capillary column (Agilent J\&W HP-5ms GC Column, $30 \mathrm{~m}, 0.25 \mathrm{~mm}, 0.25 \mu \mathrm{m}$ ). Helium was used as the carrier gas at a flow rate of $1 \mathrm{~mL} \min ^{-1}$ and injector temperature of $250^{\circ} \mathrm{C}$, and the chromatogram was obtained using the following temperature programme: $35^{\circ} \mathrm{C}$ for $1 \mathrm{~min}, 10^{\circ} \mathrm{C} \min ^{-1}$ to $250^{\circ} \mathrm{C}$ and then held at $250^{\circ} \mathrm{C}$ for $1 \mathrm{~min}$. The National Institute of Standards and Technology (NIST) MS search programme (version 2011) was used to identify the compounds based on their mass spectrum.

\section{Results}

\section{Colour and concentration changes in selenium oxyanion-amended cultures}

Each culture medium, after growth to $\mathrm{OD}_{600}$ of $0.5-0.8$, was amended separately with selenate or selenite in order to test the ability of the two methanotrophic bacteria $M s$. trichosporium OB3b and Mc. capsulatus (Bath) to reduce both selenium oxyanions. Colour changes and the selenate or selenite concentrations in each solution were monitored.

The difference in the colours of the selenite-amended solutions and corresponding spectra of the solutions is shown in Fig. 1. Similar colour change as in the Ms. trichosporium OB3b culture medium (see supplementary image S1a) was obtained when Ms. trichosporium SMDM (a derivative of Ms. trichosporium OB3b in which the genes encoding sMMO have been deleted). Changes in concentration with time at different initial selenite concentrations are shown in $\mathrm{S} 2$. Also shown in $\mathrm{S} 1 \mathrm{~b}$ is evidence that the presence of
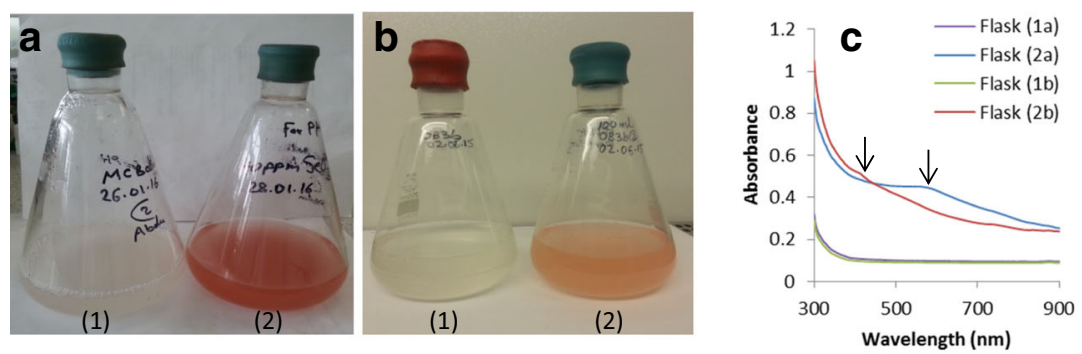

Fig. 1 Reduction of $\mathrm{SeO}_{3}{ }^{2-}$ to red $\mathrm{Se}^{0}$ by the methanotrophs $M c$. capsulatus (a) and Ms. trichosporium OB3b (b) at 48 and 72-h incubation times, respectively. Left-hand flask in each image contains no added $\mathrm{SeO}_{3}{ }^{2-}$; right-hand flask, with $\mathrm{SeO}_{3}{ }^{2-}$ added (40 and

$20 \mathrm{mg} \mathrm{L}^{-1}$, respectively). c Absorption spectra of the contents of the four flasks showing the differences in the absorption peak maximum as reflected in the solution colours 
Fig. 2 Time course of selenite reduction and \% selenite converted to elemental selenium by Mc. capsulatus (a) and Ms. trichosporium $\mathrm{OB} 3 \mathrm{~b}$ (b). Values plotted as mean \pm 1 standard deviation $(n=3)$

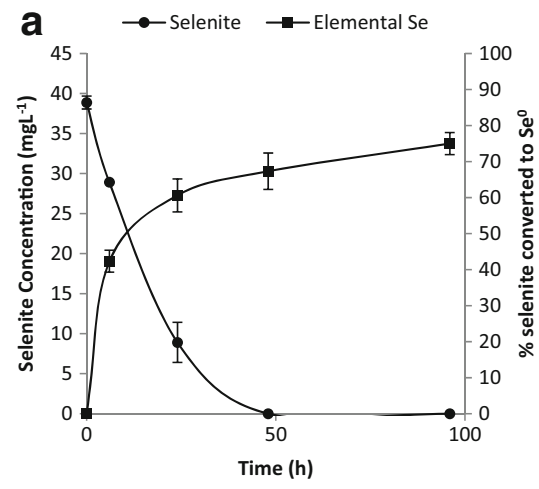

methane is essential for the reduction of selenite. No colour or concentration changes were observed in the selenate-amended cultures. Results for the determination of the selenate concentrations are shown in supplementary information S3.

\section{Transformation of selenium oxyanions and elemental selenium content of cultures}

Preliminary confirmation that the reddish/yellowish orange suspensions were primarily made up of elemental selenium was obtained by harvesting the particles and subjecting them to sample pretreatment followed by selenium determination using UV-vis spectrometry. As shown in Fig. 2, as the selenite concentrations decreased over time in each culture, the elemental selenium concentrations increased. It is noteworthy that not all the selenite in solution was converted to elemental selenium as shown by the differences in the initial selenite and the final elemental selenium concentrations for both bacteria. In cultures amended with selenate, no change was observed in the selenate concentration during the experiment. This is an indication that neither bacterium is able to reduce selenate to selenium nanoparticles or any other selenium species.

\section{Time course experiments for the formation of elemental selenium nanoparticles}

Time course experiments to show the formation of elemental selenium nanoparticles in each selenite-amended culture were performed in order to establish whether the particle sizes changed with incubation time. As shown in Fig. 3, the longer incubation time (see supplementary material S4) for either bacterium resulted in an increase in the mean selenium nanoparticle sizes, obtained by measuring 140 particles at random at the selected time (see supplementary images in S5).

\section{EXAFS and XANES measurements}

The XANES of all samples shows white line features typical for red Se as shown on top of Fig. 4a. The white line of $\mathrm{SeO}_{3}{ }^{2-}$ shown at the bottom is about $5 \mathrm{eV}$ higher in energy and coincides with the post-edge minimum of the samples and of red $\mathrm{Se}^{0}$, indicating that there are no discernible traces of Se(IV) remaining. The assignment of the spectra as due largely to red elemental selenium is also confirmed by the reconstruction of the EXAFS spectra of all samples by only one principal component, shown as red traces in Fig. $4 \mathrm{a}, \mathrm{b}$. The phase identity of red $\mathrm{Se}^{0}$ was confirmed by the Fourier transform magnitude (see Fig. 4c), which shows the two Se-Se peaks typical for the crystalline as well as the amorphous variety of red Se. The EXAFS fit shows the typical local structure, with two Se atoms at about $2.35 \AA$ and an additional Se-Se shell at $3.69 \AA$; the coordination number of this latter shell was much smaller than expected, as has been observed before for amorphous as well as for crystalline red Se (Scheinost and Charlet 2008; Scheinost et al. 2008). The EXAFS fit values also show small variations between the different samples. For strain $M c$.
Fig. 3 Time course of $\mathrm{Se}$ nanosphere growth and $\mathrm{SeO}_{3}{ }^{2-}$ reduction by Mc. capsulatus (a) and Ms. trichosporium OB3b (b). The mean selenium nanoparticle size \pm 1 standard deviation ( $n=140)$ was measured by TEM
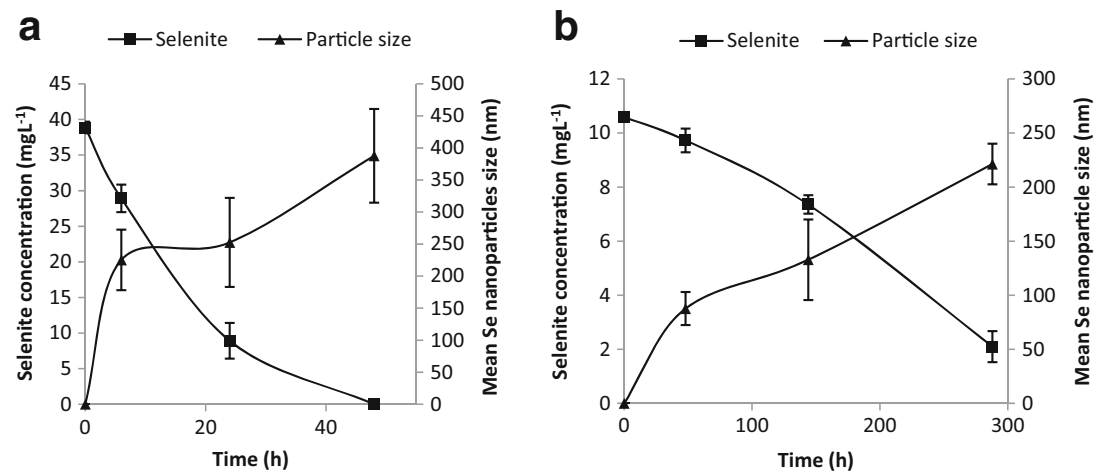
Fig. 4 Se K-edge X-ray absorption spectra of cultures of Mc. capsulatus and Ms. trichosporium and selected references. a X-ray absorption near-edge structure (XANES) spectra. b Extended X-ray absorption fine structure (EXAFS) spectra. c The corresponding Fourier transform magnitude. Experimental data are shown as black traces; the red traces in $\mathbf{b}$ and $\mathbf{c}$ are reconstructions of the experimental data by one principal component
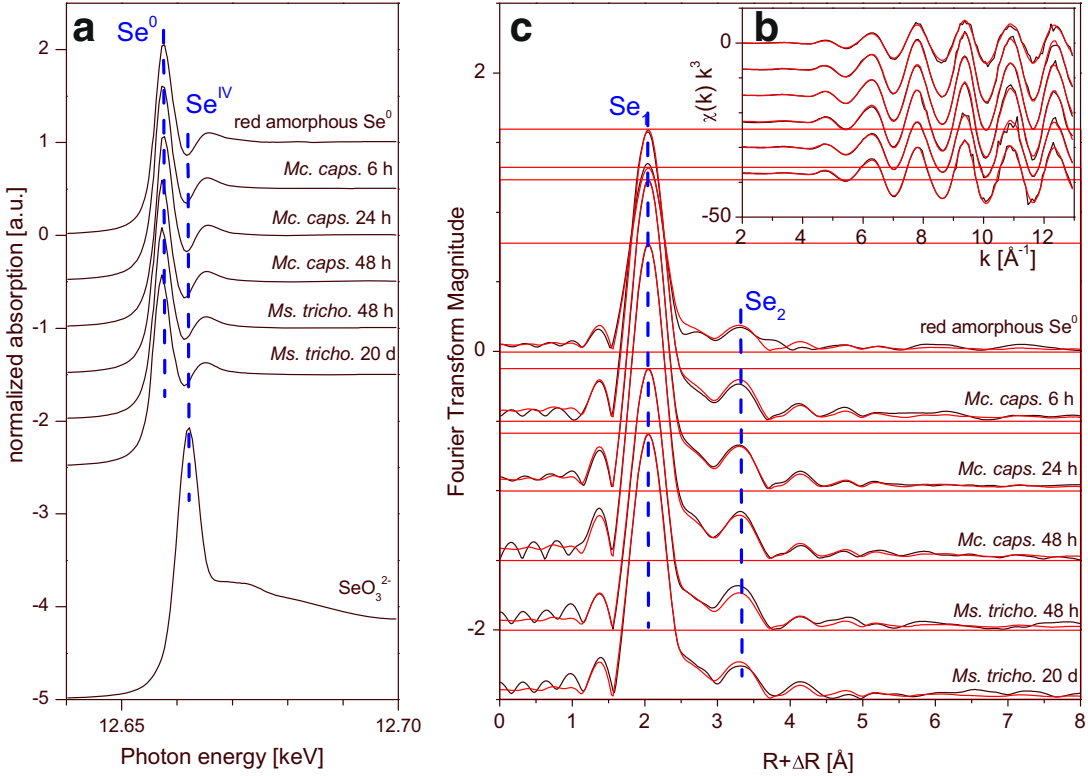

capsulatus, as well as for Ms. trichosporium, the DebyeWaller factors $\left(\sigma^{2}\right)$ of both bacteria decrease with reaction time, suggesting an increase of structural order with time (see Table 1) synchronous with the particle growth observed by TEM (see "TEM and HAADF-STEM imaging of cellassociated selenium " section).

\section{TEM and HAADF-STEM imaging of cell-associated selenium}

Electron micrographs with corresponding EDXS spectra of the cells of the two species of bacteria amended with selenite are shown in Fig. 5. The EDXS analysis of the electron-dense particles shows that they contain selenium, a trace of sulphur and phosphorus in addition to copper from the grid and possibly from the medium and Os from the cell fixing agent. The nanoparticles were spherical and in a variety of sizes. It was found that the mean elemental selenium particle sizes formed in the Mc. capsulatus medium were in the main larger than those produced by Ms. trichosporium OB3b. This is borne out in the difference in colour intensity of the selenite-amended cultures and confirmation in the differences in the two peak maximum obtained in the spectra of the two solutions. The more intense reddish colour was found in the Mc. capsulatus solutions with the larger elemental selenium particles in contrast to the yellowish orange observed in the Ms. trichosporium OB3b cultures. The mean particle sizes in the $M c$. capsulatus cultures was about $387 \mathrm{~nm}$ compared to $221 \mathrm{~nm}$ for Ms. trichosporium OB3b cultures after 48 and $288 \mathrm{~h}$ incubation, respectively.

The electron micrographs suggest that the elemental selenium nanoparticles are in extracellular space and attached to the surface of the cells suggesting that extracellular selenite reduction is followed by subsequent growth. The HAADF-STEM imaging with EDXS maps of the two bacteria is shown in Fig. 6. The electron micrograph image (white square) of Mc. capsulatus (Bath) shows the bacteria and a selenium nanoparticle. The distribution of

Table 1 Se-K edge EXAFS data of Se(IV)-reacted methanotrophs

\begin{tabular}{|c|c|c|c|c|c|c|c|c|}
\hline Sample & $\mathrm{CN}$ & $R(\AA)$ & $\sigma\left(\AA^{2}\right)$ & $\mathrm{CN}$ & $R(\AA)$ & $\sigma\left(\AA^{2}\right)$ & $\Delta E_{0}(\mathrm{eV})$ & Xres. \\
\hline Mc. capsulatus $6 \mathrm{~h}$ & $2.2 \mathrm{Se}$ & 2.35 & 0.0030 & $1.0 \mathrm{Se}$ & 3.68 & 0.0063 & 10.5 & 4.2 \\
\hline Mc. capsulatus $24 \mathrm{~h}$ & $2.1 \mathrm{Se}$ & 2.35 & 0.0024 & $0.9 \mathrm{Se}$ & 3.69 & 0.0047 & 11.1 & 2.8 \\
\hline Mc. capsulatus $48 \mathrm{~h}$ & $2.0 \mathrm{Se}$ & 2.35 & 0.0022 & $0.6 \mathrm{Se}$ & 3.69 & 0.0010 & 10.8 & 4.1 \\
\hline Ms. trichosporium OB3b $48 \mathrm{~h}$ & 1.9 Se & 2.35 & 0.0027 & $0.8 \mathrm{Se}$ & 3.69 & 0.0034 & 11.0 & 2.5 \\
\hline Ms. trichosporium OB3b 20 day & $2.1 \mathrm{Se}$ & 2.35 & 0.0032 & $0.6 \mathrm{Se}$ & 3.70 & 0.0027 & 11.5 & 3.8 \\
\hline
\end{tabular}


Fig. 5 TEM of Mc. capsulatus (a) and Ms. trichosporium $\mathrm{OB} 3 \mathrm{~b}$ (b) cultures exposed to $\mathrm{SeO}_{3}{ }^{2-}$ (20 $\mathrm{m} \mathrm{L} \mathrm{L}^{-1}$ ) and EDXS analysis in the electron-dense regions $\left(\mathrm{Se}^{0}\right.$ nanospheres)
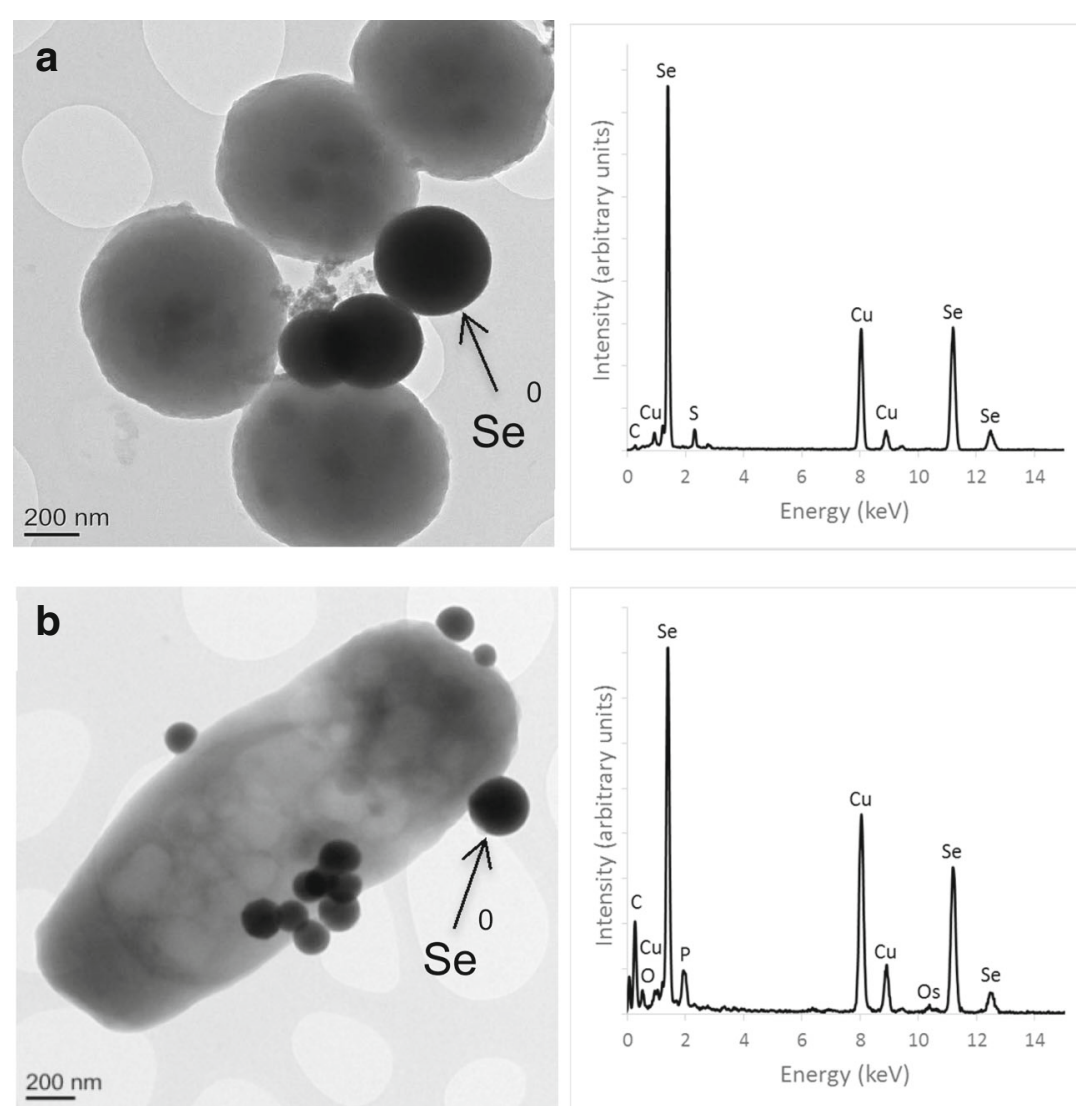

the elements $\mathrm{C}, \mathrm{O}$ and $\mathrm{P}$ map to the bacteria, and that of Se and $\mathrm{S}$ overlap in the area corresponding to the nanoparticle. Similar image and mapping of the same elements for Ms. trichosporium OB3b show that $\mathrm{C}, \mathrm{P}$ and $\mathrm{O}$ map well to the bacteria, and $\mathrm{S}$ and Se overlap, but this time, the nanoparticles are distributed all over the bacteria.
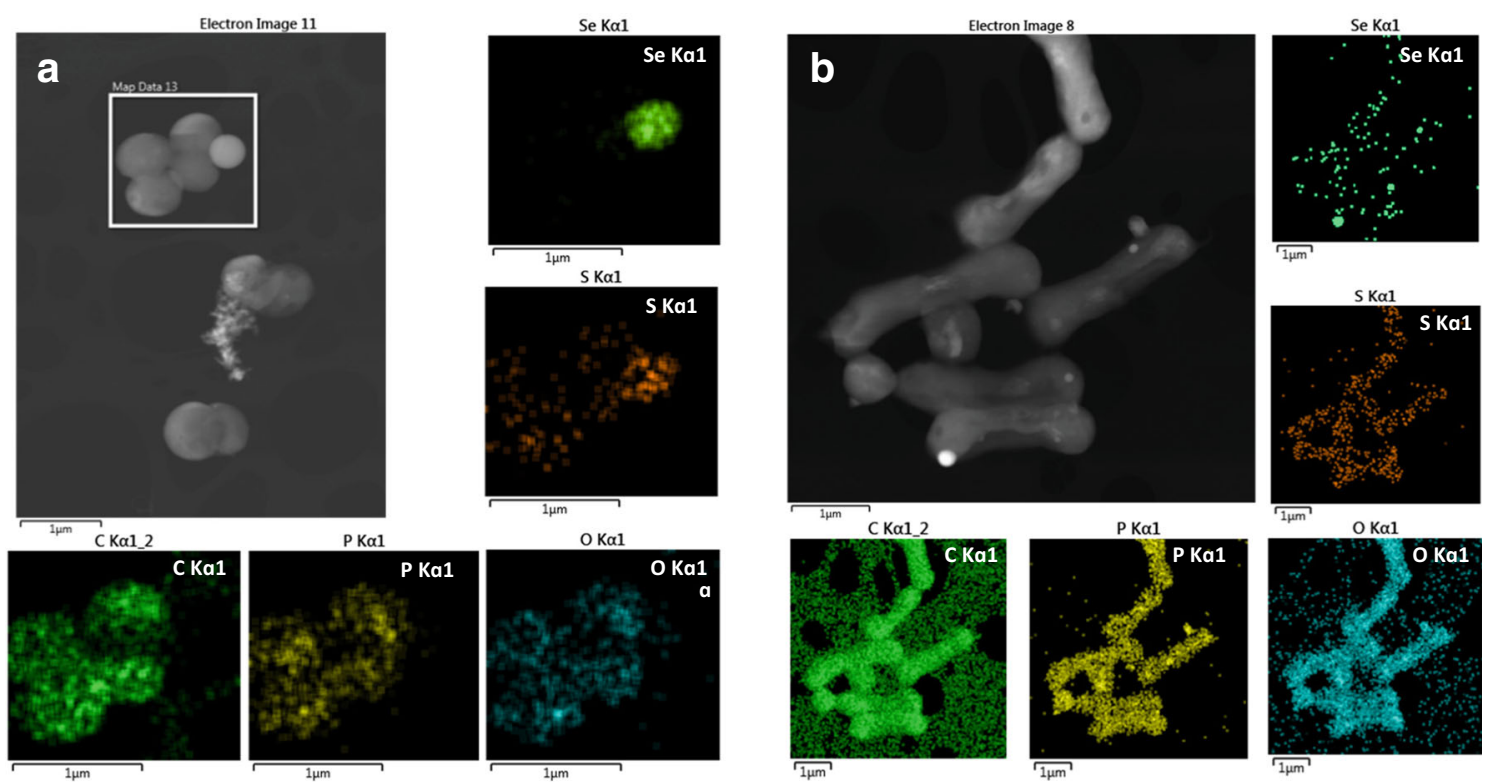

Fig. 6 HAADF-STEM imaging of Mc. capsulatus (a) and Ms. trichosporium OB3b (b) showing Se nanospheres attached to the cells with EDXS maps (generated from spectra collected from the indicated areas) of relevant elements 


\section{Confirmation of the site of selenite reduction and role of methane}

In order to confirm the site of selenite reduction and to test the hypothesis that methane gas acts as the source of electrons for the bioreduction of the selenium oxyanions, control experiments were performed with both strains from which methane was omitted, and no red colour was formed in the presence of selenite indicating that the presence of the carbon and energy source methane is needed for reduction of the selenite (see supplementary information S1b). In order to determine the cellular location of the selenite-reducing activity, experiments were performed with cell fractions: cell wall, cell membrane and cytoplasm fractions were separately amended with selenite and monitored visually (see supplementary material S6). The results showed that the red colour of elemental selenium was detected in the cell wall medium, and a weak red tinge in the cell membrane medium probably due to the traces of reductase enzyme(s) contamination, which may have diffused from the cell wall to the cell membrane (Dhanjal and Cameotra 2010).

\section{Detection and identification of volatile selenium species}

In order to detect the volatile selenium species, the headspace of the incubation flask was sampled using a syringe and injected into a GC-MS after preconcentration of the sample through a sorption tube. It was observed in preliminary experiments with selenite-amended culture medium solutions that the colour of the suspensions tended to fade with time, an indication that $\mathrm{Se}^{0}$ was probably being transformed into other selenium species. Indeed, separate experiments with harvested nanoparticles from both bacteria revealed that volatile selenium species were formed in the headspace of the flasks. Interestingly, the distribution profile of the methylated species was different compared to those formed when selenite was added to the culture medium. In the former solutions, three species, dimethyl selenide, dimethyl diselenide and dimethyl selenenyl sulphide, were detected in the headspace of both bacteria. It has been suggested by a number of investigators (Doran and Alexander 1977; Kagami et al. 2013; Chau et al. 1976; McCarthy et al. 1993; Michalke et al. 2000; Chasteen and Bentley 2003) that diverse microbes are capable of transforming selenite into volatile selenium species. In the selenite-amended cultures of either bacterium, it was observed that the volatile selenium species were detected as the red elemental selenium colour was developing. The headspace of the culture medium of both bacteria with and without selenite addition and standards were sampled and analysed for volatile selenium-containing species. GC-MS chromatograms of all the samples analysed are shown in Fig. 7, showing a variety of volatile methylated selenium and mixed seleniumsulphur species produced by both organisms. In addition to
Fig. 7 a GC-MS chromatograms of the headspace gas of the Mc. capsulatus (Bath) cultures amended with $\left(40 \mathrm{mg} \mathrm{L}^{-1}\right)$ and without selenite and that of mixed standards containing MSeH, DMSe, dimethyl disulphide (DMDS), DMSeS and DMDSe. b GC-MS chromatograms of the headspace gas of the Ms. trichosporium OB3b cultures amended with $\left(20 \mathrm{mg} \mathrm{L}^{-1}\right)$ and without selenite and that of mixed standards containing MSeH, DMSe, dimethyl disulphide (DMDS), DMSeS and DMDSe

the three previously identified methylated species, methyl selenol and methylselenoacetate were detected in the $M c$. capsulatus (Bath) headspace. In contrast, two selenium species, dimethyl diselenide and dimethyl selenenyl sulphide, were detected. Table 2 presents a summary of the volatile selenium-containing species produced when each bacterium culture is amended with selenite, biogenic selenium produced by the bacterium and commercial amorphous selenium, respectively.

\section{Discussion}

In the selenate-amended cultures, no colour or selenate concentration changes were observed, which is a strong indication that in the presence of either of these two pure strains of methantrophs, selenate is not biotransformed into elemental selenium. This finding is in contrast to that of Lai et al. (2016a), who used a biofilm microbial community in the presence of methane to show that selenate is reduced to elemental selenium. In our experiments, there were changes in the colour and oxyanion concentration only in the selenite-amended solutions. Hence, if the mixed population of methanotrophs in the study of Lai et al. had the same seleniumtransforming properties as the pure strains analysed here, the overall reaction to convert selenate to elemental selenium may have been accomplished by the combined activities of methanotrophs and other selenate-reducing, bacteria. Although there were concentration colour changes in both the $M s$. trichosporium $\mathrm{OB} 3 \mathrm{~b}$ and Mc. capsulatus (Bath) culture media to which selenite was added, the rate at which these occurred was dependant on the type of bacteria used. The slower rate of selenite reduction by the Ms. trichosporium OB3b may be linked to its slow growth rate compared with the Mc. capsulatus (Bath).

The colour change in the culture medium containing $M c$. capsulatus was rapid with perceptible reddish tinge occurring in a matter of hours and developing into an intense reddish hue in less than $24 \mathrm{~h}$. In contrast, the colour change in the $M s$. trichosporium $\mathrm{OB} 3 \mathrm{~b}$ culture medium was less intense and much slower to develop, appearing after about 2 days. However, it is noteworthy that the nanoparticles may have begun to form long before any perceptible colour change occurs in the solutions as indicated by the reduction in the selenite concentrations at the beginning of the experiments.

The results of the time course experiments (Fig. 3) provide evidence that the initial particles act as nuclei for further 

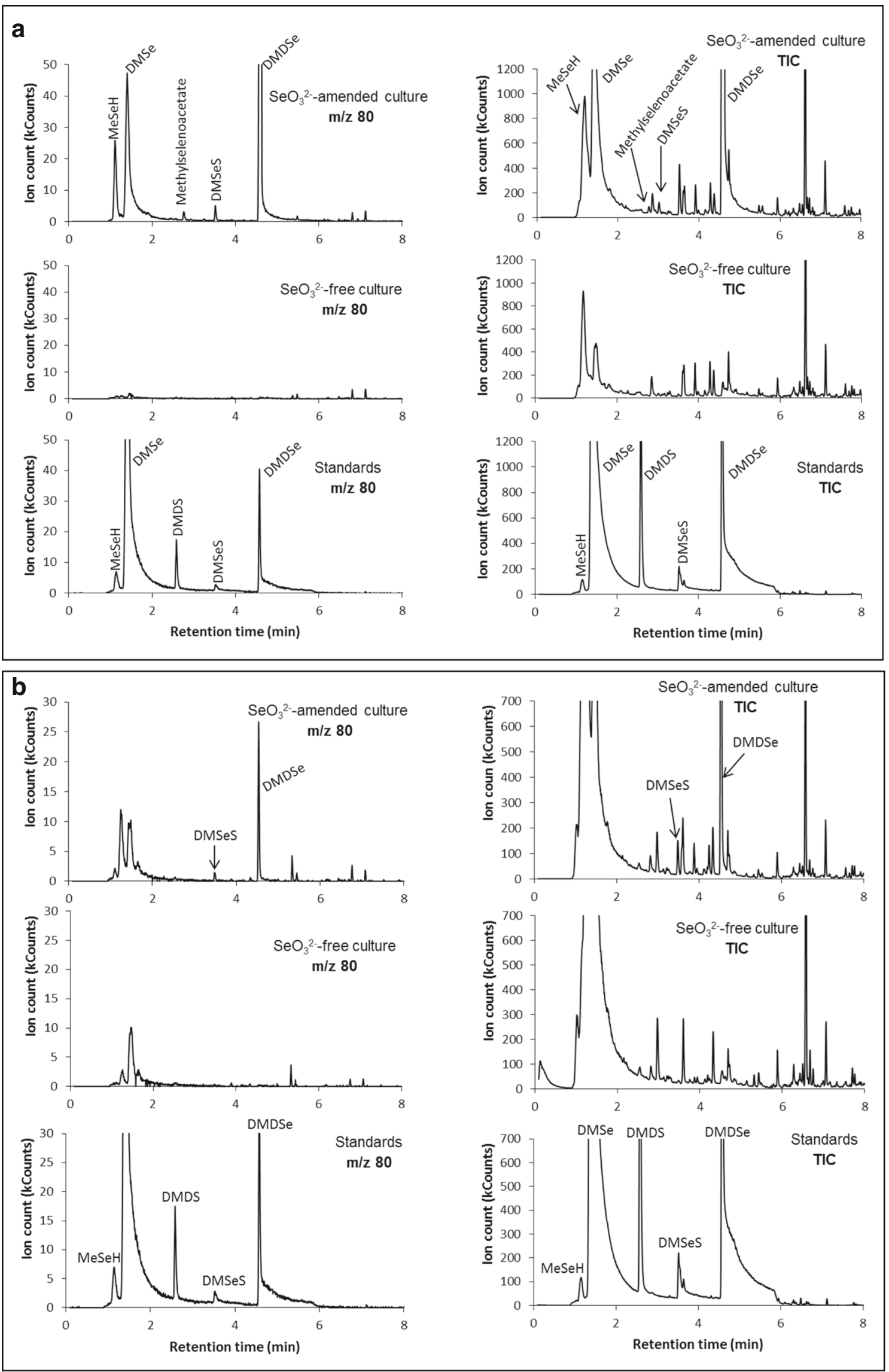
Table 2 Volatile selenium species produced by methanotrophs from different selenium-containing substrates

\begin{tabular}{llllllll}
\hline Strain & Substrate & \multicolumn{2}{l}{ Product } & & & \\
\cline { 3 - 7 } & & & DMSe & DMDSe & DMSeS & $\begin{array}{l}\text { Methyl } \\
\text { selenol }\end{array}$ & Methylselenoacetate \\
& & & & & + & + & + \\
\hline Mc. capsulatus & Selenite & + & + & + & + & - & - \\
& Bio-Se $^{0}$ & + & + & + & + & - \\
Ms. trichosporium & Che-Se $^{0}$ & + & + & + & - & - \\
OB3b & Selenite & - & + & + & - & - \\
& Bio-Se $^{0}$ & + & + & - & - & - \\
\hline
\end{tabular}

growth. In addition, it is indicative that both of these bacteria can be used to produce nanoparticles of a variety of sizes provided that there is timely intervention to stop further nanoparticle growth. TEM images taken at three different times show the growth of the particles (see supplementary information S4). Indeed, in the scheme proposed by Jain et al. (2015), the synthesis of biogenic elemental selenium (BioSeNPs) by an anaerobic granular sludge and wastewater occurs in two steps: initial reduction of selenite to elemental selenium particles either intracellularly or extracellularly followed by growth of the nanoparticles. Intracellularly produced elemental selenium nanoparticles are first coated with protein before they are expelled into extracellular space. Irrespective of the origin of the elemental selenium particles, they are invariably capped and stabilized with extracellular polymeric substances (EPSs) (Jain et al. 2015).

It can be seen from these experiments that for the production of nanoparticles of sizes less than $100 \mathrm{~nm}$, the slower reacting $M s$. trichosporium $\mathrm{OB} 3 \mathrm{~b}$ is to be preferred over the faster Mc. capsulatus. Further examination of the particles produced by both bacteria for diffraction patterns did not show evidence of any crystalline structure.

A more detailed examination of the particles with the aid of HAADF-STEM imaging and EDXS mapping provided evidence that the maps of selenium and sulphur overlap, which would suggest that both elements are present in a single structure. This is hardly surprising since the initial reactions in the previously proposed pathways for the biological reduction of selenium involve a variety of thiol group-containing compounds, which react as shown in the following equation:

$4 \mathrm{RSH}+\mathrm{H}_{2} \mathrm{SeO}_{3}=>\mathrm{RS}-\mathrm{Se}-\mathrm{SR}+R S S R+3 \mathrm{H}_{2} \mathrm{O}$

The close Se and S mapping within the EDXS images of the cultures would indicate that the pathways involving the reaction of the intermediate RS-Se-SR is likely to result in the coprecipitation of both elements. Of relevance is the observation that both sulphur and selenium are co-precipitated in the presence of sulphate-reducing bacteria (Zannoni et al. 2007). This is further evidence to indicate that there may be reactions common to the biological transformation of both elements. Abiotic and biotic reactions could together account for the coprecipitation of both elements. One example of a possible abiotic reduction reaction involving both selenium and sulphur is given by the following equation:

$\mathrm{SeO}_{3}{ }^{2-}+2 \mathrm{HS}^{-}+4 \mathrm{H}^{+}=>\mathrm{Se}^{0}+2 \mathrm{~S}^{0}+3 \mathrm{H}_{2} \mathrm{O}$

proposed by Hockin and Gadd (2003).

The results of the experiments with the cell fractions show that the reduction occurs on the cell wall of both bacteria, which is consistent with the likely extracellular location of the selenium particles that are formed. Reduction of selenite by the cell wall fractions occurred in the absence of methane. Also, since the sMMO-deleted mutant of Ms. trichosporium OB3b formed nanoparticles indistinguishable from the wildtype strain, it appears that the components of the sMMO enzyme system (including its NAD(P)H-dependent reductase) are not essential for the reduction of selenite. Since the cell wall fraction of the cells is capable of reducing selenite in the absence of added reducing agents, although the cultures as a whole require methane to perform the reaction, it seems that methane (activated either by sMMO or the particulate methane monooxygenase system) is likely the ultimate source of reducing agents, though other mediator(s) are involved in transferring the electrons to selenite (Smith and Murrell 2011).

Identification of the selenium-containing species in the headspace of the cultures was achieved by matching the retention times of the standards together with mass spectra information stored in the instrument NIST library database. Using this approach, it was possible to detect methyl selenol $\left(\mathrm{CH}_{3} \mathrm{SeH}, \mathrm{MSeH}\right)$, methylselenoacetate $\left(\mathrm{C}_{3} \mathrm{H}_{6} \mathrm{OSe}\right)$, dimethyl selenide $\left(\mathrm{CH}_{3} \mathrm{SeCH}_{3}\right.$, DMSe $)$, dimethyl diselenide $\left(\mathrm{CH}_{3} \mathrm{SeSeCH}_{3}\right.$, DMDSe $)$ and dimethyl selenenyl sulphide $\left(\mathrm{CH}_{3} \mathrm{SeSCH}_{3}\right.$, DMSeS $)$ in the headspace of selenite-amended Mc. capsulatus culture medium. In contrast, only two volatile selenium-containing 
species, DMDSe and DMSeS, were detected in the headspace of the selenite-amended Ms. trichosporium culture. It is noteworthy that with both culture media, these selenium species were detected soon after selenite addition. Results of experiments with the harvested nanoparticles clearly show that these are required for the formation of volatile selenium species, but also, other species are directly formed through other possible pathways as suggested by Challenger (1945) and Reamer and Zoller (1980). The manner in which the methylation of selenium may link to the one-carbon central metabolism of the methanotrophs remains to be established.

The results presented above clearly indicate that the pure strains of the methanotrophic bacteria Mc. capsulatus and Ms. trichosporium OB3b are able to reduce selenite but not selenate to produce elemental selenium and volatile selenium species. The formation of elemental selenium appears to be mainly an extracellular process, probably accomplished indirectly with electrons derived from methane. It is probable that reducing agents containing sulfhydryl groups on the cell wall plays a key role in the bioreduction process of selenite. This opens the possibility that methanotrophs (which are widespread across diverse environments) may play a significant role in the global selenium cycle. The results also suggest that these bacteria may be useful in preparing selenium nanoparticles of a range of sizes for biotechnological applications. Much remains to be determined about the pathway of selenium biotransformations in methanotrophs, though it appears that elemental selenium may not necessarily be an intermediate on the pathway to the formation of all volatile selenium species.

Acknowledgements A.S.E. gratefully acknowledges financial support from the Libyan Government for a Ph.D. scholarship. The European Synchrotron Radiation Facility (ESRF) is acknowledged for the X-ray absorption spectroscopy measurements. We thank Michael Cox (Sheffield Hallam University) for assistance with the HPLC-ICP-MS measurements and Jonathan Foster (Sheffield Hallam University) for assistance with the GC-MS analysis.

\section{Compliance with ethical standards}

Conflict of interest The authors declare that they have no conflict of interest.

Ethical approval This article does not contain any studies with human participants or animals performed by any of the authors.

Open Access This article is distributed under the terms of the Creative Commons Attribution 4.0 International License (http:// creativecommons.org/licenses/by/4.0/), which permits unrestricted use, distribution, and reproduction in any medium, provided you give appropriate credit to the original author(s) and the source, provide a link to the Creative Commons license, and indicate if changes were made.

\section{References}

Al Hasin A, Gurman SJ, Murphy LM, Perry A, Smith TJ, Gardiner PH (2009) Remediation of chromium (VI) by a methane-oxidizing bacterium. Environ Sci Technol 44:400-405

Ankudinov AL, Rehr J (1997) Relativistic calculations of spin-dependent X-ray-absorption spectra. Phys Rev B 56:R1712

Bebien M, Chauvin JP, Adriano JM, Grosse S, Vermeglio A (2001) Effect of selenite on growth and protein synthesis in the phototrophic bacterium Rhodobacter sphaeroides. Appl Environ Microbiol 67: $4440-4447$

Birringer M, Pilawa S, Flohé L (2002) Trends in selenium biochemistry. Nat Prod Rep 19:693-718

Biswas KC, Barton LL, Tsui WL, Shuman K, Gillespie J, Eze CS (2011) A novel method for the measurement of elemental selenium produced by bacterial reduction of selenite. J Microbiol Methods 86: 140-144

Boden R, Murrell JC (2011) Response to mercury (II) ions in Methylococcus capsulatus (Bath). FEMS Microbiol Lett 324:106110

Borodina E, Nichol T, Dumont MG, Smith TJ, Murrell JC (2007) Mutagenesis of the "leucine gate" to explore the basis of catalytic versatility in soluble methane monooxygenase. Appl Environ Microbiol 73:6460-6467

Challenger F (1945) Biological methylation. Chem Rev 36:315-361

Chasteen TG (1993) Confusion between dimethyl selenenyl sulfide and dimethyl selenone released by bacteria. Appl Organomet Chem 7: 335-342

Chasteen TG, Bentley R (2003) Biomethylation of selenium and tellurium: microorganisms and plants. Chem Rev 103:1-26

Chau YK, Wong PT, Silverberg BA, Luxon PL, Bengert GA (1976) Methylation of selenium in the aquatic environment. Science 192: $1130-1131$

Dhanjal S, Cameotra SS (2010) Aerobic biogenesis of selenium nanospheres by Bacillus cereus isolated from coalmine soil. Microb Cell Factories 9:52

Doran JW, Alexander M (1977) Microbial transformations of selenium. Appl Environ Microbiol 33:31-37

Dungan R, Frankenberger W (1999) Microbial transformations of selenium and the bioremediation of seleniferous environments. Bioremediat J 3:171-188

Eswayah AS, Smith TJ, Gardiner PH (2016) Microbial transformations of selenium species of relevance to bioremediation. Appl Environ Microbiol 82:4848-4859

Hanson RS, Hanson TE (1996) Methanotrophic bacteria. Microbiol Rev 60:439-471

Haug A, Graham RD, Christophersen OA, Lyons GH (2007) How to use the world's scarce selenium resources efficiently to increase the selenium concentration in food. Microb Ecol Health Dis 19:209 228

Heider J, Bock A (1993) Selenium metabolism in micro-organisms. Adv Microb Physiol 35:71-109

Hockin SL, Gadd GM (2003) Linked redox precipitation of sulfur and selenium under anaerobic conditions by sulfate-reducing bacterial biofilms. Appl Environ Microbiol 69:7063-7072

Jain R, Jordan N, Weiss S, Foerstendorf H, Heim K, Kacker R, Hübner R, Kramer H, Van Hullebusch ED, Farges F (2015) Extracellular polymeric substances govern the surface charge of biogenic elemental selenium nanoparticles. Environ Sci Technol 49:1713-1720

Jiang H, Chen Y, Jiang P, Zhang C, Smith TJ, Murrell JC, Xing X (2010) Methanotrophs: multifunctional bacteria with promising applications in environmental bioengineering. Biochem Eng J 49:277-288

Johansson L, Gafvelin G, Arnér ES (2005) Selenocysteine in proteinsproperties and biotechnological use. Biochim Biophys Acta Gen Subj 1726:1-13 
Kagami T, Narita T, Kuroda M, Notaguchi E, Yamashita M, Sei K, Soda S, Ike M (2013) Effective selenium volatilization under aerobic conditions and recovery from the aqueous phase by Pseudomonas stutzeri NT-I. Water Res 47:1361-1368

Kalyuzhnaya MG, Puri AW, Lidstrom ME (2015) Metabolic engineering in methanotrophic bacteria. Metab Eng 29:142-152

Klonowska A, Heulin T, Vermeglio A (2005) Selenite and tellurite reduction by Shewanella oneidensis. Appl Environ Microbiol 71:56075609

Lai C, Wen L, Shi L, Zhao K, Wang Y, Yang X, Rittmann BE, Zhou C, Tang Y, Zheng P (2016a) Selenate and nitrate bioreductions using methane as the electron donor in a membrane biofilm reactor. Environ Sci Technol 50:10179-10186

Lai C, Zhong L, Zhang Y, Chen J, Wen L, Shi L, Sun Y, Ma F, Rittmann BE, Zhou C (2016b) Bio-reduction of chromate in a methane-based membrane biofilm reactor. Environ Sci Technol

Lenz M, Lens PN (2009) The essential toxin: the changing perception of selenium in environmental sciences. Sci Total Environ 407:3620 3633

McCarthy S, Chasteen T, Marshall M, Fall R, Bachofen R (1993) Phototrophic bacteria produce volatile, methylated sulfur and selenium compounds. FEMS Microbiol Lett 112:93-97

Michalke K, Wickenheiser EB, Mehring M, Hirner AV, Hensel R (2000) Production of volatile derivatives of metal(loid)s by microflora involved in anaerobic digestion of sewage sludge. Appl Environ Microbiol 66:2791-2796

Patching S, Gardiner R (1999) Recent developments in selenium metabolism and chemical speciation: a review. J Trace Elem Med Biol 13: 193-214

Qin H, Zhu J, Liang L, Wang M, Su H (2013) The bioavailability of selenium and risk assessment for human selenium poisoning in high-Se areas, China. Environ Int 52:66-74

Reamer DC, Zoller WH (1980) Selenium biomethylation products from soil and sewage sludge. Science 208:500-502

Ressler T (1998) WinXAS: a program for X-ray absorption spectroscopy data analysis under MS-Windows. J Synchrotron Radiat 5:118-122
Rossberg A, Reich T, Bernhard G (2003) Complexation of uranium (VI) with protocatechuic acid - application of iterative transformation factor analysis to EXAFS spectroscopy. Anal Bioanal Chem 376: $631-638$

Roy AB, Trudinger PA (1970) The biochemistry of inorganic compounds of sulphur. Cambridge University Press

Scheinost AC, Charlet L (2008) Selenite reduction by mackinawite, magnetite and siderite: XAS characterization of nanosized redox products. Environ Sci Technol 42:1984-1989

Scheinost AC, Kirsch R, Banerjee D, Fernandez-Martinez A, Zaenker H, Funke H, Charlet L (2008) X-ray absorption and photoelectron spectroscopy investigation of selenite reduction by Fe II-bearing minerals. J Contam Hydrol 102:228-245

Shamberger R (2012) Biochemistry of selenium. Springer Science \& Business Media

Smith TJ, Murrell JC (2011) Mutagenesis of soluble methane monooxygenase. Methods Enzymol 495:135

Smith TJ, Foster SJ (1995) Characterization of the involvement of two compensatory autolysins in mother cell lysis during sporulation of Bacillus subtilis 168. J Bacteriol 177:3855-3862

Smith T, Murrell JC (2009) Methanotrophy/methane oxidation. Encylopedia of Microbiology. Elsevier, Oxford, pp 293-298

Stadtman T (1991) Biosynthesis and function of selenocysteinecontaining enzymes. J Biol Chem 266:16257-16260

Switzer Blum J, Burns Bindi A, Buzzelli J, Stolz JF, Oremland RS (1998) Bacillus arsenicoselenatis, sp. nov., and Bacillus selenitireducens, sp. nov.: two haloalkaliphiles from Mono Lake, California that respire oxyanions of selenium and arsenic. Arch Microbiol 171:19-30

Switzer Blum J, Stolz JF, Oren A, Oremland RS (2001) Selenihalanaerobacter shriftii gen. nov., sp. nov., a halophilic anaerobe from Dead Sea sediments that respires selenate. Arch Microbiol 175:208-219

Webb S (2005) SIXpack: a graphical user interface for XAS analysis using IFEFFIT. Phys Scripta 2005:1011

Zannoni D, Borsetti F, Harrison JJ, Turner RJ (2007) The bacterial response to the chalcogen metalloids Se and Te. Adv Microb Physiol 53:1-312 